Claremont Colleges

Scholarship@ Claremont

CMC Faculty Publications and Research

CMC Faculty Scholarship

$1-1-2013$

\title{
Near-optimal compressed sensing guarantees for anisotropic and isotropic total variation minimization
}

Deanna Needell

Claremont McKenna College

Rachel Ward

University of Texas at Austin

\section{Recommended Citation}

Needell, Deanna and Ward, Rachel, "Near-optimal compressed sensing guarantees for anisotropic and isotropic total variation minimization" (2013). CMC Faculty Publications and Research. Paper 318.

http://scholarship.claremont.edu/cmc_fac_pub/318

This Technical Report is brought to you for free and open access by the CMC Faculty Scholarship at Scholarship @ Claremont. It has been accepted for inclusion in CMC Faculty Publications and Research by an authorized administrator of Scholarship @ Claremont. For more information, please contact scholarship@cuc.claremont.edu. 


\title{
Near-optimal compressed sensing guarantees for anisotropic and isotropic total variation minimization
}

\author{
Deanna Needell and Rachel Ward
}

\begin{abstract}
Consider the problem of reconstructing a multidimensional signal from partial information, as in the setting of compressed sensing. Without any additional assumptions, this problem is ill-posed. However, for signals such as natural images or movies, the minimal total variation estimate consistent with the measurements often produces a good approximation to the underlying signal, even if the number of measurements is far smaller than the ambient dimensionality. Recently, guarantees for two-dimensional images $\boldsymbol{x} \in \mathbb{C}^{N^{2}}$ were established. This paper extends these theoretical results to signals $\boldsymbol{x} \in \mathbb{C}^{N^{d}}$ of arbitrary dimension $d \geq 2$ and to both the anisotropic and isotropic total variation problems. To be precise, we show that a multidimensional signal $\boldsymbol{x} \in \mathbb{C}^{N^{d}}$ can be reconstructed from $\mathcal{O}\left(s d \log \left(N^{d}\right)\right)$ linear measurements $\boldsymbol{y}=\boldsymbol{A} \boldsymbol{x}$ using total variation minimization to within a factor of the best $s$-term approximation of its gradient. The reconstruction guarantees we provide are necessarily optimal up to polynomial factors in the spatial dimension $d$ and a logarithmic factor in the signal dimension $N^{d}$. The proof relies on bounds in approximation theory concerning the compressibility of wavelet expansions of bounded-variation functions.
\end{abstract}

\section{INTRODUCTION}

Compressed sensing (CS) is a new signal processing methodology where signals are acquired in compressed form as undersampled linear measurements. The applications of CS are abundant, ranging

Claremont McKenna College, 850 Columbia Ave, Claremont CA, 91711, email: dneedell@cmc.edu.

University of Texas at Austin, 2515 Speedway, Austin, Texas, 77842, email: rward@math.utexas.edu. R. Ward has been supported in part by a Donald D. Harrington Faculty Fellowship, Alfred P. Sloan Research Fellowship, and DOD-Navy grant N00014-12-1-0743. 
from radar and error correction to many areas of image processing [18]. The underlying assumption that makes such acquisition and reconstruction possible is that most natural signals are sparse or compressible. We say that a signal $\boldsymbol{x} \in \mathbb{C}^{p}$ is $s$-sparse when

$$
\|\boldsymbol{x}\|_{0} \stackrel{\text { def }}{=}|\operatorname{supp}(\boldsymbol{x})| \leq s \ll p .
$$

Compressible signals are those which are well-approximated by sparse signals. More generally, a signal $\boldsymbol{x} \in \mathbb{C}^{p}$ is said to be $s$-sparse with respect to a basis $\boldsymbol{B}$ when $\boldsymbol{x}$ can be represented as a linear combination of $s$ atoms from $\boldsymbol{B}$. In the CS framework, we acquire $m \ll p$ nonadaptive linear measurements of the form

$$
\boldsymbol{y}=\mathcal{M}(\boldsymbol{x})+\boldsymbol{\xi}
$$

where $\mathcal{M}: \mathbb{C}^{p} \rightarrow \mathbb{C}^{m}$ is an appropriate linear operator and $\boldsymbol{\xi}$ is vector modeling additive noise. The theory of CS [21], [9], [22] ensures that under suitable assumptions on the measurement operator $\mathcal{M}$, a sufficiently compressible signal can be accurately approximated by the signal of minimal $\ell_{1}$-norm consistent with the measurements,

$$
\hat{\boldsymbol{x}} \in \underset{\boldsymbol{w}}{\operatorname{argmin}}\|\boldsymbol{w}\|_{1} \quad \text { such that }\|\mathcal{M}(\boldsymbol{w})-\boldsymbol{y}\|_{2} \leq \varepsilon,
$$

where $\|\boldsymbol{w}\|_{1}=\sum_{i}\left|w_{i}\right|$ and $\|\boldsymbol{w}\|_{2}=\left(\sum_{i}\left|w_{i}\right|^{2}\right)^{1 / 2}$ denote the standard $\ell_{1}$ and Euclidean norms, $\varepsilon$ bounds the noise level $\|\boldsymbol{\xi}\|_{2} \leq \varepsilon$, and argmin denotes the set of minimizers (if a unique minimizer exists this set will contain one element). The program $\left(L_{1}\right)$ may be cast as a second order cone program (SOCP) and can be solved efficiently using standard convex programming methods (see e.g. [3], [17]).

To guarantee robust recovery of compressible signals via $\left(L_{1}\right)$, Candès and Tao introduced in [9] the restricted isometry property (RIP) for a measurement operator $\mathcal{M}$.

Definition 1. A linear operator $\mathcal{M}: \mathbb{C}^{p} \rightarrow \mathbb{C}^{m}$ is said to have the restricted isometry property (RIP) of order $s \in \mathbb{N}$ and level $\delta \in(0,1)$ if

$$
(1-\delta)\|\boldsymbol{x}\|_{2}^{2} \leq\|\mathcal{M}(\boldsymbol{x})\|_{2}^{2} \leq(1+\delta)\|\boldsymbol{x}\|_{2}^{2} \quad \text { for all s-sparse } \boldsymbol{x} \in \mathbb{C}^{p} \text {. }
$$

Many distributions of random matrices of dimension $m \times p$ are known to generate RIP matrices of order $s$ and level $\delta \leq c<1$ for $m \approx \delta^{-2} s(\log p)^{4}$. Note that here and throughout we have used the notation $u \approx v$ (analogously $u \lesssim v$ ) to indicate that there exists some absolute constant $C>0$ such that $u=C v(u \leq C v)$. Representative families of random matrices include randomly subsampled rows 
from the discrete Fourier transform [46] or from a bounded orthonormal system more generally [46], [44], [42], [43], [5], and randomly-generated circulant matrices [28]. Moreover, a matrix whose entries are independent and identical (i.i.d.) realizations of a properly-normalized subgaussian random variable will have the RIP with probability exceeding $1-e^{-c m}$ once $m \approx \delta^{-2} s \log (p / s)$ [10], [36], [46], [1].

Candès, Romberg, and Tao [8] showed that when the measurement operator $\mathcal{M}$ has the RIP of order $\mathcal{O}(s)$ and sufficiently small constant $\delta$, the program $\left(L_{1}\right)$ recovers an estimation $\hat{\boldsymbol{x}}$ to $\boldsymbol{x}$ that satisfies the error bound

$$
\|\hat{\boldsymbol{x}}-\boldsymbol{x}\|_{2} \leq C\left(\frac{\left\|\boldsymbol{x}-\boldsymbol{x}_{\boldsymbol{s}}\right\|_{1}}{\sqrt{s}}+\varepsilon\right),
$$

where $\boldsymbol{x}_{\boldsymbol{s}}$ denotes the best $s$-sparse approximation to the signal $\boldsymbol{x}$. Using properties about Gel'fand widths of the $\ell_{1}$ ball due to Kashin [26] and Garnaev-Gluskin [23], this is the optimal minimax reconstruction rate for $\ell_{1}$-minimization using $m \approx s \log (p / s)$ nonadaptive linear measurements. Due to the rotationalinvariance of an RIP matrix with randomized column signs [29], a completely analogous theory holds for signals that are compressible with respect to a known orthonormal basis or tight frame $D$ by replacing $\boldsymbol{w}$ with $\boldsymbol{D}^{*} \boldsymbol{w}$ inside the $\ell_{1}$-norm of the minimization problem $\left(L_{1}\right)$ [6], [32].

\section{A. Imaging with $C S$}

Natural images are highly compressible with respect to their gradient representation. For an image $\boldsymbol{x} \in \mathbb{C}^{N^{2}}$ one defines its discrete directional derivatives by

$$
\begin{aligned}
\boldsymbol{x}_{u}: \mathbb{C}^{N \times N} \rightarrow \mathbb{C}^{(N-1) \times N}, & \left(\boldsymbol{x}_{u}\right)_{j, k}=\boldsymbol{x}_{j+1, k}-\boldsymbol{x}_{j, k} \\
\boldsymbol{x}_{v}: \mathbb{C}^{N \times N} \rightarrow \mathbb{C}^{N \times(N-1)}, & \left(\boldsymbol{x}_{v}\right)_{j, k}=\boldsymbol{x}_{j, k+1}-\boldsymbol{x}_{j, k} .
\end{aligned}
$$

The discrete gradient transform $\nabla: \mathbb{C}^{N^{2}} \rightarrow \mathbb{C}^{N \times N \times 2}$ is defined in terms of the directional derivatives,

$$
(\boldsymbol{\nabla} \boldsymbol{x})_{j, k} \stackrel{\text { def }}{=} \begin{cases}\left(\left(\boldsymbol{x}_{u}\right)_{j, k},\left(\boldsymbol{x}_{v}\right)_{j, k}\right), & 1 \leq j \leq N-1, \quad 1 \leq k \leq N-1 \\ \left(0,\left(\boldsymbol{x}_{v}\right)_{j, k}\right), & j=N, \quad 1 \leq k \leq N-1 \\ \left(\left(\boldsymbol{x}_{u}\right)_{j, k}, 0\right), & k=N, \quad 1 \leq j \leq N-1 \\ (0,0), & j=k=N\end{cases}
$$

The $\ell_{1}$-norm of the discrete gradient defines a seminorm for the space $\mathbb{C}^{N^{2}}$, often referred to as the anisotropic total variation seminorm and denoted by

$$
\|\boldsymbol{x}\|_{T V} \stackrel{\text { def }}{=}\|\boldsymbol{\nabla} \boldsymbol{x}\|_{1}
$$


The isotropic total variation norm results from the choice of $\left(\boldsymbol{x}_{u}\right)_{j, k}+i\left(\boldsymbol{x}_{v}\right)_{j, k}$ instead of $\left(\left(\boldsymbol{x}_{u}\right)_{j, k},\left(\boldsymbol{x}_{v}\right)_{j, k}\right)$ in the definition of the discrete gradient. In the isotropic case, $\|\boldsymbol{x}\|_{T V}$ becomes the sum of terms

$$
\left|\left(\boldsymbol{x}_{u}\right)_{j, k}+i\left(\boldsymbol{x}_{v}\right)_{j, k}\right|=\left(\left(\boldsymbol{x}_{u}\right)_{j, k}^{2}+\left(\boldsymbol{x}_{v}\right)_{j, k}^{2}\right)^{1 / 2}
$$

Due to the gradient sparsity of natural images, it should not be surprising that the total variation minimization program

$$
\hat{\boldsymbol{x}} \in \underset{\boldsymbol{z}}{\operatorname{argmin}}\|\boldsymbol{z}\|_{T V} \quad \text { such that } \quad\|\mathcal{M}(\boldsymbol{z})-\boldsymbol{y}\|_{2} \leq \varepsilon
$$

is often used for image and other signal reconstruction, and the literature now provides much theoretical understanding in the general setting. Properties of TV minimizers in inverse problems have been studied in the discrete and continuous settings [2], [39], [20], [47], [45], [40], [12], [13], and convergence rates of stability measures for TV have also been established (see e.g. [4], [24]). In the setting of compressed sensing and more broadly in other imaging applications, it has been used for denoising, deblurring, and inpainting (see e.g. [8], [11], [7], [41], [14], [33], [34], [31], [38], [35], [25], [27], [49], [37] and the references therein). We consider here the TV problem in the compressed sensing setting.

While (TV) is similar to the $\ell_{1}$-minimization program $\left(L_{1}\right)$, the RIP-based theoretical guarantees for $\left(L_{1}\right)$ do not directly translate to recovery guarantees for (TV) because the gradient map $\boldsymbol{z} \rightarrow \boldsymbol{\nabla} \boldsymbol{z}$ is not well-conditioned on the orthogonal complement of $\operatorname{ker}(\boldsymbol{\nabla})$. In fact, viewed as an invertible operator over mean-zero images, the condition number of the gradient map is proportional to the image side length $N .{ }^{1}$ Recovery guarantees for anisotropic $(T V)$ in the compressed sensing setting were nevertheless obtained in [37] for two-dimensional images $\boldsymbol{x} \in \mathbb{C}^{N^{2}}$ by showing that the gradient map is well-conditioned when restricted to signals lying in the null space of a matrix with the restricted isometry property. The following theorem holds for any image $\boldsymbol{x} \in \mathbb{C}^{N^{2}}$.

Theorem A (from [37]). There are choices of linear operators $\mathcal{M}: \mathbb{C}^{N^{2}} \rightarrow \mathbb{C}^{m}$ with $m \approx s \log \left(N^{2} / s\right)$ for which the following holds for any image $\boldsymbol{x} \in \mathbb{C}^{N^{2}}$ : Given noisy measurements $\boldsymbol{y}=\mathcal{M}(\boldsymbol{x})+\boldsymbol{\xi}$ with noise level $\|\boldsymbol{\xi}\|_{2} \leq \varepsilon$, the total-variation minimizing signal

$$
\hat{\boldsymbol{x}} \in \underset{\boldsymbol{z}}{\operatorname{argmin}}\|\boldsymbol{z}\|_{T V} \quad \text { such that }\|\mathcal{M}(\boldsymbol{z})-\boldsymbol{y}\|_{2} \leq \varepsilon
$$

\footnotetext{
${ }^{1}$ One sees that the norm of $\boldsymbol{\nabla}$ is a constant whereas the norm of its inverse is proportional to $N$ (one can observe this scaling, for example, by noting it is obtained by the image whose entries are constant).
} 
satisfies the error bound

$$
\|\boldsymbol{x}-\hat{\boldsymbol{x}}\|_{2} \leq C \log \left(N^{2} / s\right)\left(\frac{\left\|\boldsymbol{\nabla} \boldsymbol{x}-(\boldsymbol{\nabla} \boldsymbol{x})_{s}\right\|_{1}}{\sqrt{s}}+\varepsilon\right),
$$

where here and throughout, $\boldsymbol{z}_{s}$ denotes the best s-term approximation to the array $\boldsymbol{z}$.

In words, the total-variation minimizer estimates $\boldsymbol{x}$ to within a factor of the noise level and best $s$-term approximation of its gradient. The bound in (8) is optimal up to the logarithmic factor $\log \left(N^{2} / s\right)$.

The contribution of this paper is to extend Theorem A to multidimensional signals $\boldsymbol{x} \in \mathbb{C}^{N^{d}}$ of arbitrary dimension $d \geq 2$. We show that the signal $\hat{\boldsymbol{x}} \in \mathbb{C}^{N^{d}}$ of minimal ( $d$-dimensional) total variation seminorm consistent with $m \approx s d \log \left(N^{d}\right)$ appropriately-chosen linear measurements $\boldsymbol{y}=\mathcal{M}(\boldsymbol{x})+\boldsymbol{\xi}$ will approximate $\boldsymbol{x}$ to within a factor of the noise level and the best $s$-term approximation to the $(d$ dimensional) discrete gradient of $\boldsymbol{x}$, modulo a single logarithmic factor in the signal dimension $N^{d}$. In addition, we generalize the theory to other types of total variation norms, which include both the isotropic and anisotropic versions.

Our proof rests on extending the Sobolev inequalities for random subspaces from [37] to higherdimensional signal structures, using bounds of Cohen, Dahmen, Daubechies, and DeVore in [15] on the compressibility of wavelet representations for functions of bounded variation. Unfortunately these bounds, and hence our results for total variation, do not hold in dimension $d=1$. See [48] for results for one-dimensional total variation under assumptions other than the RIP.

\section{B. Organization}

The article is organized as follows. In Section II we recall relevant background material on the multidimensional total variation seminorm and multidimensional orthonormal wavelet transform. Section III states our main result: total variation minimization provides stable signal recovery for signals of arbitrary dimension $d \geq 2$. The proof of this result will occupy the remainder of the paper; in Section IV we prove that the signal gradient is recovered stably, while in Section $\mathrm{V}$ we pass from stable gradient recovery to stable signal recovery using the strengthened Sobolev inequalities for random subspaces. The proofs of propositions and theorems used along the way are contained in the appendix.

\section{PRELIMINARIES FOR MULTIDIMENSIONAL SigNAL ANALYSiS}

The setting for this article is the space $\mathbb{C}^{N^{d}}$ of multidimensional arrays of complex numbers, consisting of elements

$$
\boldsymbol{x}=\left(x_{\boldsymbol{\alpha}}\right)_{\alpha \in[N]^{d}} \in \mathbb{C}^{N^{d}}, \quad \boldsymbol{\alpha} \equiv\left(\alpha_{1}, \alpha_{2}, \ldots, \alpha_{d}\right) \in[N]^{d},
$$


where $[N]^{d}=\{1,2, \ldots, N\}^{d}$. Note that here and throughout we use the convention that vectors such as $\boldsymbol{x}$ are boldface and their scalar counterparts such as $x_{\boldsymbol{\alpha}}$ are normal typeface. The space $\mathbb{C}^{N^{d}}$ is a Hilbert space using the standard inner product

$$
\langle\boldsymbol{x}, \boldsymbol{y}\rangle=\sum_{\boldsymbol{\alpha} \in[N]^{d}} x_{\boldsymbol{\alpha}} \cdot \bar{y}_{\boldsymbol{\alpha}}
$$

where $\bar{y}$ denotes the conjugate of $y$. It is isometric to the subspace $\Sigma_{N}$ of $L_{2}\left([0,1)^{d}\right)^{2}$ consisting of functions which are constant over cubes of side length $N^{-1}$,

$$
\Sigma_{N}=\left\{f \in L_{2}\left([0,1)^{d}\right), f(\boldsymbol{u})=f_{\boldsymbol{\alpha}}, \quad \frac{\alpha_{i}-1}{N} \leq u_{i}<\frac{\alpha_{i}}{N}\right\} .
$$

For $\boldsymbol{x}=\left(x_{\boldsymbol{\alpha}}\right) \in \mathbb{C}^{N^{d}}$, the isometry is provided by identifying $f_{\boldsymbol{\alpha}}=f_{\boldsymbol{\alpha}}^{\boldsymbol{x}}=N^{d / 2} x_{\boldsymbol{\alpha}}$. More generally, we denote by $\|\boldsymbol{x}\|_{p}=\left(\sum_{\boldsymbol{\alpha} \in[N]^{d}}\left|x_{\boldsymbol{\alpha}}\right|^{p}\right)^{1 / p}$ the entrywise $\ell_{p}$-norm of the signal $\boldsymbol{x}$.

For $\ell=1,2, \ldots d$, the discrete derivative of $\boldsymbol{x}$ in the direction of $r_{\ell}$ is the array $\boldsymbol{x}_{r_{\ell}} \in \mathbb{C}^{N^{\ell-1} \times(N-1) \times N^{d-\ell}}$ defined component-wise by

$$
\left(\boldsymbol{x}_{r_{\ell}}\right)_{\boldsymbol{\alpha}} \stackrel{\text { def }}{=} x_{\left(\alpha_{1}, \alpha_{2}, \ldots, \alpha_{\ell}+1, \ldots, \alpha_{d}\right)}-x_{\left(\alpha_{1}, \alpha_{2}, \ldots, \alpha_{\ell}, \ldots \alpha_{d}\right)},
$$

and we define the $d$-dimensional discrete gradient transform $\nabla: \mathbb{C}^{N^{d}} \rightarrow \mathbb{C}^{d \times N^{d}}$ through its components

$$
(\boldsymbol{\nabla} \boldsymbol{x})_{\ell, \boldsymbol{\alpha}} \stackrel{\text { def }}{=} \begin{cases}\left(\boldsymbol{x}_{r_{\ell}}\right)_{\boldsymbol{\alpha}}, & \alpha_{\ell} \leq N-1, \\ 0, & \text { else }\end{cases}
$$

Now let $B_{1}, B_{2}, \ldots, B_{K}$ be a partition of $\{1, \ldots, d\}$ into blocks of equal size $b=d / K=\left|B_{i}\right|$. The generalized $d$-dimensional total-variation seminorm is the mixed $\ell_{1}-\ell_{2}$ norm of the $d$-dimensional discrete gradient,

$$
\|\boldsymbol{x}\|_{T V} \stackrel{\text { def }}{=} \sum_{\boldsymbol{\alpha} \in[N]^{d}} \sum_{i=1}^{k}\left(\sum_{\ell \in B_{i}}\left(\boldsymbol{x}_{r_{\ell}}\right)_{\boldsymbol{\alpha}}^{2}\right)^{1 / 2} .
$$

Note then that the anisotropic total variation seminorm results from the choice of $B_{i}=\{i\}$ with $b=1$, and the isotropic version from the choice of $B_{1}=\{1, \ldots, d\}$ with $b=d$. To treat these versions simultaneously, throughout the remainder of the article we write $\|\cdot\|_{T V}$ to denote the generalized total variation norm and distinguish between types through the choice of $b$.

\footnotetext{
${ }^{2}$ Recall that $f \in L_{2}(Q)$ if $\int_{Q}|f(u)|^{2} d u<\infty$, and $L_{2}(Q)$ is a Hilbert space equipped with the inner product $\langle f, g\rangle=$ $\int_{Q} f(u) \cdot \bar{g}(u) d u$
} 
A linear operator $\mathcal{A}: \mathbb{C}^{N^{d}} \rightarrow \mathbb{C}^{p}$ can be represented as a sequence of multidimensional arrays $\mathcal{A}=\left(\boldsymbol{a}_{k}\right)$. The linear operation $\boldsymbol{y}=\mathcal{A}(\boldsymbol{x})$ has component-wise action

$$
y_{k}=[\mathcal{A}(\boldsymbol{x})]_{k}=\left\langle\boldsymbol{a}_{k}, \boldsymbol{x}\right\rangle
$$

where the inner product between multidimensional arrays is defined in (9). A linear operator $\mathcal{A}: \mathbb{C}^{N^{d}} \rightarrow$ $\mathbb{C}^{N^{d}}$ can be expressed similarly through its components $y_{\boldsymbol{\alpha}}=[\mathcal{A}(\boldsymbol{x})]_{\boldsymbol{\alpha}}=\left\langle\boldsymbol{a}_{\boldsymbol{\alpha}}, \boldsymbol{x}\right\rangle$.

Finally, we introduce the row direct sum operation for concatenating linear operators: if $\mathcal{A}: \mathbb{C}^{N^{d}} \rightarrow \mathbb{C}^{r_{1}}$ and $\mathcal{B}: \mathbb{C}^{N^{d}} \rightarrow \mathbb{C}^{r_{2}}$ then $\mathcal{M}=\mathcal{A} \oplus_{r} \mathcal{B}$ is the linear operator from $\mathbb{C}^{N^{d}}$ to $\mathbb{C}^{r_{1}+r_{2}}$ with component arrays $\mathcal{M}=\left(\boldsymbol{m}_{k}\right)_{k=1}^{r_{1}+r_{2}}$ given by

$$
\boldsymbol{m}_{k}= \begin{cases}\boldsymbol{a}_{k}, & 1 \leq k \leq r_{1}, \\ \boldsymbol{b}_{k-r_{1}}, & 1+r_{1} \leq k \leq r_{1}+r_{2}\end{cases}
$$

Alternatively, the column direct sum operation for concatenating linear operators $\mathcal{A}: \mathbb{C}^{N^{d}} \rightarrow \mathbb{C}^{p}$ and $\mathcal{B}: \mathbb{C}^{N^{d}} \rightarrow \mathbb{C}^{p}$ yields the linear operator $\mathcal{M}=\mathcal{A} \oplus_{c} \mathcal{B}: \mathbb{C}^{2 \times N^{d}} \rightarrow \mathbb{C}^{p}$ with component arrays $\mathcal{M}=\left(\boldsymbol{m}_{k}\right)_{k=1}^{p}$ given by

$$
\left(\boldsymbol{m}_{k}\right)_{\ell, \boldsymbol{\alpha}}= \begin{cases}\left(\boldsymbol{a}_{k}\right)_{\boldsymbol{\alpha}}, & \ell=1 \\ \left(\boldsymbol{b}_{k}\right)_{\boldsymbol{\alpha}}, & \ell=2\end{cases}
$$

\section{A. The multidimensional Haar wavelet transform}

The Haar wavelet transform provides a sparsifying basis for natural signals such as images and movies, and is closely related to the discrete gradient. The Haar transform plays an important role in our analysis for passing from the $\ell_{1}$-theory of compressed sensing to theory for total variation minimization. For a comprehensive introduction to wavelets, we refer the reader to [19].

The (continuous) multidimensional Haar wavelet basis is derived from a tensor-product representation of the univariate Haar basis, which forms an orthonormal system for square-integrable functions on the unit interval and consists of the constant function

$$
h^{0}(t)= \begin{cases}1 & 0 \leq t<1 \\ 0, & \text { otherwise }\end{cases}
$$

the step function

$$
h^{1}(t)= \begin{cases}1 & 0 \leq t<1 / 2 \\ -1 & 1 / 2 \leq t<1\end{cases}
$$

and dyadic dilations and translations of the step function,

$$
h_{j, k}(t)=2^{j / 2} h^{1}\left(2^{j} t-k\right) ; \quad j \in \mathbb{N}, \quad 0 \leq k<2^{j} .
$$


The Haar basis for the higher dimensional space $L_{2}(Q)$ of square-integrable functions on the unit cube $Q=[0,1)^{d}$ consists of tensor-products of the univariate Haar wavelets. Concretely, for $V=\{0,1\}^{d}-\{0\}^{d}$ and $\boldsymbol{e}=\left(e_{1}, e_{2}, \ldots, e_{d}\right) \in V$, we define the multivariate functions

$$
h^{e}(\boldsymbol{u})=\prod_{e_{i}} h^{e_{i}}\left(u_{i}\right)
$$

The orthonormal Haar system on $L_{2}(Q)$ is then comprised of the constant function along with all functions of the form

$$
h_{j, k}^{e}(\boldsymbol{u})=2^{j d / 2} h^{e}\left(2^{j} \boldsymbol{u}-k\right), \quad \boldsymbol{e} \in V, \quad j \geq 1, \quad k \in \mathbb{Z}^{d} \cap 2^{j} Q .
$$

The discrete multidimensional Haar transform is derived from the continuous construction via the isometric identification (10) between $\mathbb{C}^{N^{d}}$ and $\Sigma_{N} \subset L_{2}(Q)$ : defining

$$
\boldsymbol{h}_{j, k, \boldsymbol{e}}(\boldsymbol{\alpha})=N^{-d / 2} h_{j, k}^{\boldsymbol{e}}(\boldsymbol{\alpha} / N), \quad \boldsymbol{\alpha} \in[N]^{d},
$$

the matrix product computing the discrete Haar transform can be expressed as $\mathcal{H} \boldsymbol{x}=\left(\left\langle\boldsymbol{h}_{j, k, \boldsymbol{e}}, \boldsymbol{x}\right\rangle\right)_{j, k, \boldsymbol{e}}$. Note that with this normalization, the transform is orthonormal.

\section{B. Gradient versus wavelet sparsity}

Since the multivariate Haar transform $\mathcal{H}: \mathbb{C}^{N^{d}} \rightarrow \mathbb{C}^{N^{d}}$ is orthonormal, standard results in compressed sensing (e.g. [11]) guarantee that by minimizing the $\ell_{1}$-norm of the conjugate transpose or inverse Haar transform (note that $\mathcal{H}^{*}=\mathcal{H}^{-1}$ ),

$$
\tilde{\boldsymbol{x}} \in \underset{\boldsymbol{z}}{\operatorname{argmin}}\left\|\mathcal{H}^{*}(\boldsymbol{z})\right\|_{1} \quad \text { such that } \quad\|\mathcal{M}(\boldsymbol{z})-\boldsymbol{y}\|_{2} \leq \varepsilon
$$

a multidimensional signal can be reconstructed from $m \geq C s \log \left(N^{d} / s\right)$ measurements to within a factor of its best $s$-term approximation in the Haar basis. A straightforward calculation verifies that a signal which is $s$-sparse with respect to the discrete gradient is $s \log (N)$-sparse with respect to the Haar transform. Moreover, this relationship between gradient and wavelet sparsity is stable, as can be seen from the following corollary of results from [15] on the decay of wavelet representations associated to functions of bounded variation:

Proposition 2 (Corollary of Theorem 1.1 from [15]). There is a universal constant $C>0$ such that the following holds for any $\boldsymbol{x} \in \mathbb{C}^{N^{d}}$ in dimension $d \geq 2$ : if the Haar transform coefficients $\boldsymbol{c}=\mathcal{H}(\boldsymbol{x})$ are 
partitioned by their support into blocks $\boldsymbol{c}_{j, k}=\left(\left\langle\boldsymbol{h}_{j, k, e}, \boldsymbol{x}\right\rangle\right)_{e \in V}$ of cardinality $\left|\boldsymbol{c}_{j, k}\right|=2^{d}-1$, then the coefficient block of kth largest $\ell_{2}$-norm, denoted by $c_{(k)}$, has $\ell_{2}$-norm bounded by

$$
\left\|\boldsymbol{c}_{(k)}\right\|_{2} \leq C \frac{\sqrt{b}\|\boldsymbol{x}\|_{T V}}{k \cdot 2^{d / 2-1}}
$$

Proposition 2, whose derivation from Theorem 1.1 of [15] is outlined in the appendix, will provide us the link necessary to prove robust recovery via total variation using orthonormality properties of the wavelet transform. This proof will occupy the remainder of the present article.

\section{THE MAIN RESULT}

Our main result concerns near-optimal recovery guarantees for multidimensional total variation minimization from compressed measurements. Recall that a linear operator $\mathcal{A}: \mathbb{C}^{N^{d}} \rightarrow \mathbb{C}^{p}$ is said to have the restricted isometry property (RIP) of order $s$ and level $\delta \in(0,1)$ when

$$
(1-\delta)\|\boldsymbol{x}\|_{2}^{2} \leq\|\mathcal{A}(\boldsymbol{x})\|_{2}^{2} \leq(1+\delta)\|\boldsymbol{x}\|_{2}^{2} \quad \text { for all } s \text {-sparse } \boldsymbol{x} \in \mathbb{C}^{N^{d}} \text {. }
$$

A linear operator $\mathcal{A}=\left(\boldsymbol{a}_{k}\right): \mathbb{C}^{N^{d}} \rightarrow \mathbb{C}^{p}$ satisfies the RIP if and only if the $p \times N^{d}$ matrix $\boldsymbol{A}$ whose $k$ th row consists of the unraveled entries of the $k$ th multidimensional array $\boldsymbol{a}_{k}$ satisfies the classical RIP, (1), and so without loss of generality we treat both definitions of the RIP as equivalent.

For our main result it will be convenient to define for a multidimensional array $\boldsymbol{a} \in \mathbb{C}^{N^{\ell-1} \times(N-1) \times N^{d-\ell}}$ the associated arrays $\boldsymbol{a}_{0_{\ell}} \in \mathbb{C}^{N^{d}}$ and $\boldsymbol{a}^{0_{\ell}} \in \mathbb{C}^{N^{d}}$ obtained by concatenating a block of zeros to the beginning and end of $\boldsymbol{a}$ oriented in the $\ell$ th direction:

$$
\left(\boldsymbol{a}^{0_{\ell}}\right)_{\boldsymbol{\alpha}}= \begin{cases}0, & \alpha_{\ell}=1 \\ a_{\alpha_{1}, \ldots, \alpha_{\ell}-1, \ldots, \alpha_{d}}, & 2 \leq \alpha_{\ell} \leq N\end{cases}
$$

and

$$
\left(\boldsymbol{a}_{0_{\ell}}\right)_{\boldsymbol{\alpha}}= \begin{cases}0, & \alpha_{\ell}=N \\ a_{\alpha_{1}, \ldots, \alpha_{\ell}, \ldots, \alpha_{d}}, & 1 \leq \alpha_{\ell} \leq N-1\end{cases}
$$

The following lemma relating gradient measurements with $\boldsymbol{a}$ to signal measurements with $\boldsymbol{a}^{0_{\ell}}$ and $\boldsymbol{a}_{0_{\ell}}$ can be verified by direct algebraic manipulation and thus the proof is omitted.

Lemma 3. Given $\boldsymbol{x} \in \mathbb{C}^{N^{d}}$ and $\boldsymbol{a} \in \mathbb{C}^{N^{\ell-1} \times(N-1) \times N^{d-\ell}}$,

$$
\left\langle\boldsymbol{a}, \boldsymbol{x}_{r_{\ell}}\right\rangle=\left\langle\boldsymbol{a}^{0_{\ell}}, \boldsymbol{x}\right\rangle-\left\langle\boldsymbol{a}_{0_{\ell}}, \boldsymbol{x}\right\rangle,
$$

where the directional derivative $\boldsymbol{x}_{r_{\ell}}$ is defined in (11). 
For a linear operator $\mathcal{A}=\left(\boldsymbol{a}_{k}\right): \mathbb{C}^{N^{\ell-1} \times(N-1) \times N^{d-\ell}} \rightarrow \mathbb{C}^{m}$ we define the operators $\mathcal{A}^{0_{\ell}}: \mathbb{C}^{N^{d}} \rightarrow \mathbb{C}^{m}$ and $\mathcal{A}_{0_{\ell}}: \mathbb{C}^{N^{d}} \rightarrow \mathbb{C}^{m}$ as the sequences of arrays $\left(\boldsymbol{a}_{k}^{0_{k}}\right)_{k=1}^{m}$ and $\left(\boldsymbol{a}_{0_{k}}\right)_{k=1}^{m}$, respectively. From Lemma 21, $\mathcal{A}\left(\boldsymbol{x}_{r_{\ell}}\right)=\mathcal{A}^{0_{\ell}}(\boldsymbol{x})-\mathcal{A}_{0_{\ell}}(\boldsymbol{x})$.

We are now prepared to state our main result which shows that total variation minimization yields stable recovery of $N^{d}$-dimensional signals from RIP measurements.

Main Theorem. Let $N=2^{n}$. Fix integers $p$ and $q$, and let $\mathcal{H}: \mathbb{C}^{N^{d}} \rightarrow \mathbb{C}^{N^{d}}$ be the orthonormal Haar wavelet transform, and let $\mathcal{A}: \mathbb{C}^{N^{d}} \rightarrow \mathbb{C}^{p}$ be such that the composite operator $\mathcal{A} \mathcal{H}^{*}: \mathbb{C}^{N^{d}} \rightarrow \mathbb{C}^{p}$ has the restricted isometry property of order $2 s$ and level $\delta<1$. Let $\mathcal{B}_{1}, \mathcal{B}_{2}, \ldots, \mathcal{B}_{d}$ with $\mathcal{B}_{j}: \mathbb{C}^{N^{d-1}(N-1)} \rightarrow \mathbb{C}^{q}$ be such that $\mathcal{B}=\mathcal{B}_{1} \oplus_{c} \mathcal{B}_{2} \oplus_{c} \cdots \oplus_{c} \mathcal{B}_{d}: \mathbb{C}^{N^{d-1}(N-1)} \rightarrow \mathbb{C}^{d q}$ has the restricted isometry property of order 5 sb and level $\delta<1 / 3$. Set $m=2 d q+p$, and consider the linear operator $\mathcal{M}: \mathbb{C}^{N^{d}} \rightarrow \mathbb{C}^{m}$ given by

$$
\mathcal{M}=\mathcal{A} \oplus_{r}\left[\mathcal{B}_{1}\right]^{0_{1}} \oplus_{r}\left[\mathcal{B}_{1}\right]_{0_{1}} \oplus_{r} \cdots \oplus_{r}\left[\mathcal{B}_{\ell}\right]^{0_{\ell}} \oplus_{r}\left[\mathcal{B}_{\ell}\right]_{0_{\ell}} \oplus_{r} \cdots \oplus_{r}\left[\mathcal{B}_{d}\right]^{0_{d}} \oplus_{r}\left[\mathcal{B}_{d}\right]_{0_{d}}
$$

The following holds for all $\boldsymbol{x} \in \mathbb{C}^{N^{d}}$ : From noisy measurements $\boldsymbol{y}=\mathcal{M}(\boldsymbol{x})+\boldsymbol{\xi}$ with noise level $\|\boldsymbol{\xi}\|_{2} \leq \varepsilon$, the solution to (TV) (total variation with parameter b) satisfies:

i) $\|\boldsymbol{\nabla}(\boldsymbol{x}-\hat{\boldsymbol{x}})\|_{2} \lesssim \frac{\left\|\boldsymbol{\nabla} \boldsymbol{x}-(\boldsymbol{\nabla} \boldsymbol{x})_{s}\right\|_{1 b}}{\sqrt{s}}+\sqrt{d} \varepsilon$,

ii) $\|\boldsymbol{x}-\hat{\boldsymbol{x}}\|_{T V} \lesssim\left\|\boldsymbol{\nabla} \boldsymbol{x}-(\boldsymbol{\nabla} \boldsymbol{x})_{s}\right\|_{1 b}+\sqrt{s d} \varepsilon$,

iii) $\|\boldsymbol{x}-\hat{\boldsymbol{x}}\|_{2} \lesssim \log \left(N^{d}\right)\left(\frac{\sqrt{b}\left\|\boldsymbol{\nabla} \boldsymbol{x}-(\boldsymbol{\nabla} \boldsymbol{x})_{s}\right\|_{1 b}}{\sqrt{s}}+\sqrt{b d} \varepsilon\right)$, where $\|\boldsymbol{z}\|_{1 b}:=\sum_{\alpha \in[N]^{d}} \sum_{i=1}^{k}\left(\sum_{\ell \in B_{i}}(\boldsymbol{z})_{\ell, \boldsymbol{\alpha}}^{2}\right)^{1 / 2}$ denotes the mixed $\ell_{1}-\ell_{2}$ norm.

Applying the main result to the isotropic $(b=d)$ and anisotropic cases $(b=1)$ yield the following corollaries.

Corollary 4 (Isotropic total variation). Under the notation and assumptions of the Main Theorem, the minimizer of isotropic total variation (TV) satisfies:

i) $\|\boldsymbol{\nabla}(\boldsymbol{x}-\hat{\boldsymbol{x}})\|_{2} \lesssim \frac{\left\|\boldsymbol{\nabla} \boldsymbol{x}-(\boldsymbol{\nabla} \boldsymbol{x})_{s}\right\|_{1,2}}{\sqrt{s}}+\sqrt{d} \varepsilon$,

ii) $\|\boldsymbol{x}-\hat{\boldsymbol{x}}\|_{T V} \lesssim\left\|\boldsymbol{\nabla} \boldsymbol{x}-(\boldsymbol{\nabla} \boldsymbol{x})_{s}\right\|_{1,2}+\sqrt{s d} \varepsilon$,

iii) $\|\boldsymbol{x}-\hat{\boldsymbol{x}}\|_{2} \lesssim \log \left(N^{d}\right)\left(\frac{\sqrt{b}\left\|\boldsymbol{\nabla} \boldsymbol{x}-(\boldsymbol{\nabla} \boldsymbol{x})_{s}\right\|_{1,2}}{\sqrt{s}}+d \varepsilon\right)$,

where $\|\boldsymbol{z}\|_{1,2}:=\sum_{\alpha \in[N]^{d}}\left(\sum_{\ell=1}^{d}(\boldsymbol{z})_{\ell, \boldsymbol{\alpha}}^{2}\right)^{1 / 2}$ denotes the standard mixed $\ell_{1}-\ell_{2}$ norm. 
Corollary 5 (Anisotropic total variation). Under the notation and assumptions of the Main Theorem, the minimizer of anisotropic total variation (TV) satisfies:
i) $\|\boldsymbol{\nabla}(\boldsymbol{x}-\hat{\boldsymbol{x}})\|_{2} \lesssim \frac{\left\|\boldsymbol{\nabla} \boldsymbol{x}-(\boldsymbol{\nabla} \boldsymbol{x})_{s}\right\|_{1}}{\sqrt{s}}+\sqrt{d} \varepsilon$,
ii) $\|\boldsymbol{x}-\hat{\boldsymbol{x}}\|_{T V} \lesssim\left\|\boldsymbol{\nabla} \boldsymbol{x}-(\boldsymbol{\nabla} \boldsymbol{x})_{s}\right\|_{1}+\sqrt{s d} \varepsilon$,
iii) $\|\boldsymbol{x}-\hat{\boldsymbol{x}}\|_{2} \lesssim \log \left(N^{d}\right)\left(\frac{\left\|\boldsymbol{\nabla} \boldsymbol{x}-(\boldsymbol{\nabla} \boldsymbol{x})_{s}\right\|_{1}}{\sqrt{s}}+\sqrt{d} \varepsilon\right)$.

\section{Remarks.}

1. A number of $m \approx s d \log \left(N^{d}\right)$ i.i.d. and properly normalized Gaussian measurements can be used to construct the measurement operator $\mathcal{M}$ which, with high probability, satisfies the required RIP conditions of the theorem [1], [46]. From this number $m$ of measurements, the error guarantees $i$ and $i i$ are optimal up to the factor of $\sqrt{d}$ on the noise level, and the error guarantee $i i i$ is optimal up to logarithmic factors in the signal dimension $N^{d}$. We emphasize here that the specific construction of the measurement ensemble is likely only an artifact of the proof, and that more general RIP measurements are likely possible. See also [30] for results using Fourier measurements (for $d=2$ ).

2. When $d=2$, the main theorem recovers the total variation stability guarantee of [37] up to a $\log (1 / s)$ term. Note that this term is lost only because in higher dimensions the proof requires a blocking of the wavelet coefficients, and is thus likely only an artifact of the proof. It may be improved by a more efficient blocking strategy and by writing in terms of $p$ in (29) of the proof. We write the bound as-is for simplicity.

3. The requirement of sidelength $N=2^{n}$ is not an actual restriction, as signals with arbitrary sidelength $N$ can be extended via reflections across each dimension to a signal of side-length $N=2^{n}$ without increasing the total variation by more than a factor of $2^{d}$. This requirement again seems to be only an artifact of the proof and one need not perform such changes in practice.

4. Note that we actually prove something stronger than Corollary 4. Indeed, the term $\left\|\boldsymbol{\nabla} \boldsymbol{x}-(\boldsymbol{\nabla} \boldsymbol{x})_{s}\right\|_{1,2}$ in the bounds can be replaced with $\left\|(\boldsymbol{\nabla} \boldsymbol{x})_{R^{c}}\right\|_{1,2}$ for a set $R$ with $|R|=s d$. See the proof of the Main Theorem below for details.

We now turn to the proof of the main theorem. We follow the method of proof introduced in [37], proving stable gradient recovery and then translating these guarantees to stable signal recovery via Sobolev inequalities for incoherent subspaces. 


\section{STABLE GRADIENT RECOVERY}

In this section we prove statements $(i)$ and $(i i)$ of the main theorem concerning stable gradient recovery, using standard results in the $\ell_{1}$ theory of compressed sensing combined with a summation by parts "trick" provided by Lemma 3.

Recall that when a signal obeys a tube and cone constraint we can bound the norm of the entire signal, as in [11]. We refer the reader to Section A.1 of [37] for a complete proof.

Proposition 6. Suppose that $\mathcal{B}$ is a linear operator satisfying the restricted isometry property of order 5 sb and level $\delta<1 / 3$, and suppose that the signal $\boldsymbol{h}$ satisfies a tube constraint

$$
\|\mathcal{B}(\boldsymbol{h})\|_{2} \leq \sqrt{2 d} \varepsilon
$$

Suppose further that using the notation of the Main Theorem, for a subset $R$ of cardinality $|R| \leq s b$ (with complement $R^{c}$ ), $\boldsymbol{h}$ satisfies a cone-constraint

$$
\left\|\boldsymbol{h}_{R^{c}}\right\|_{1 b} \leq\left\|\boldsymbol{h}_{R}\right\|_{1 b}+\sigma
$$

Then

$$
\|\boldsymbol{h}\|_{2} \lesssim \frac{\sigma}{\sqrt{s}}+\sqrt{d} \varepsilon
$$

and

$$
\|\boldsymbol{h}\|_{1 b} \lesssim \sigma+\sqrt{s d} \varepsilon
$$

The proof of Proposition 6 is a generalization of results in [8] and included in the appendix for completeness. Using Proposition 6 and the RIP assumptions on the operator $\mathcal{B}$, stable gradient recovery (i) and (ii) reduce to proving that the discrete gradient of the residual signal error satisfies the tube and cone constraints.

Proof: (Main Theorem, statements (i) and (ii).) Let $\boldsymbol{v}=\boldsymbol{x}-\hat{\boldsymbol{x}}$ be the residual error, and set $h=\boldsymbol{\nabla} \boldsymbol{v}$. Let $\mathcal{B}=\left(\mathcal{B}_{k}\right)$. Then we have

Cone Constraint. Let $R=\left\{(\ell, \boldsymbol{\alpha}):\left(\sum_{\ell \in B_{i}}\left(\boldsymbol{x}_{r_{\ell}}\right)_{\boldsymbol{\alpha}}^{2}\right)^{1 / 2}\right.$ is one of the $s$ largest over all choices of $B_{i}$ and $\left.\boldsymbol{\alpha}\right\}$ denote the set of pairs $(\ell, \boldsymbol{\alpha})$ corresponding to the largest magnitudes in $\boldsymbol{\nabla} \boldsymbol{x}$ so that if $\left(\ell_{1}, \boldsymbol{\alpha}\right) \in R$, for some $\ell_{1} \in B_{i}$ then $(\ell, \boldsymbol{\alpha}) \in R$ for all other $\ell \in B_{i}$. In particular, observe that $|R|=s b$. Since $\hat{\boldsymbol{x}}=\boldsymbol{x}-\boldsymbol{v}$ is a minimizer of (TV) and $\boldsymbol{x}$ satisfies the feasibility constraint 
in (TV), $\|\boldsymbol{\nabla} \hat{\boldsymbol{x}}\|_{1 b} \leq\|\boldsymbol{\nabla} \boldsymbol{x}\|_{1 b}$ and by the reverse triangle inequality,

$$
\begin{aligned}
\left\|(\boldsymbol{\nabla} \boldsymbol{x})_{R}\right\|_{1 b}-\left\|\boldsymbol{h}_{R}\right\|_{1 b}-\left\|(\boldsymbol{\nabla} \boldsymbol{x})_{R^{c}}\right\|_{1 b} & +\left\|\boldsymbol{h}_{R^{c}}\right\|_{1 b} \\
& \leq\left\|(\boldsymbol{\nabla} \boldsymbol{x})_{R}-\boldsymbol{h}_{R}\right\|_{1 b}+\left\|(\boldsymbol{\nabla} \boldsymbol{x})_{R^{c}}-\boldsymbol{h}_{R^{c}}\right\|_{1 b} \\
& =\|\boldsymbol{\nabla} \hat{\boldsymbol{x}}\|_{1 b} \\
& \leq\|\boldsymbol{\nabla} \boldsymbol{x}\|_{1 b} \\
& =\left\|(\boldsymbol{\nabla} \boldsymbol{x})_{R}\right\|_{1 b}+\left\|(\boldsymbol{\nabla} \boldsymbol{x})_{R^{c}}\right\|_{1 b} .
\end{aligned}
$$

This yields the cone constraint

$$
\left\|\boldsymbol{h}_{R^{c}}\right\|_{1 b} \leq\left\|\boldsymbol{h}_{R}\right\|_{1 b}+2\left\|(\boldsymbol{\nabla} \boldsymbol{x})_{R^{c}}\right\|_{1 b} \leq\left\|\boldsymbol{h}_{R}\right\|_{1 b}+2\left\|\boldsymbol{\nabla} \boldsymbol{x}-(\boldsymbol{\nabla} \boldsymbol{x})_{s}\right\|_{1 b} .
$$

Tube constraint. Recall that $\boldsymbol{v}=\boldsymbol{x}-\hat{\boldsymbol{x}}$. Since both $\boldsymbol{x}$ and $\hat{\boldsymbol{x}}$ are feasible solutions to (TV), Jensen's inequality gives

$$
\begin{aligned}
\|\mathcal{M}(\boldsymbol{v})\|_{2}^{2} & \leq 2\|\mathcal{M}(\boldsymbol{x})-\boldsymbol{y}\|_{2}^{2}+2\|\mathcal{M}(\hat{\boldsymbol{x}})-\boldsymbol{y}\|_{2}^{2} \\
& \leq 4 \varepsilon^{2}
\end{aligned}
$$

By Lemma 3, we have for each component operator $\mathcal{B}_{j}$,

$$
\mathcal{B}_{j}\left(\boldsymbol{v}_{r_{j}}\right)=\left[\mathcal{B}_{j}\right]^{0_{j}}(\boldsymbol{v})-\left[\mathcal{B}_{j}\right]_{0_{j}}(\boldsymbol{v})
$$

Then $\mathcal{B}(\boldsymbol{\nabla} \boldsymbol{v})=\sum_{j=1}^{d} \mathcal{B}_{j}\left(\boldsymbol{v}_{r_{j}}\right)$, (where we assume that $\boldsymbol{\nabla} \boldsymbol{v}$ is ordered appropriately) and

$$
\begin{aligned}
\|\mathcal{B}(\boldsymbol{\nabla} \boldsymbol{v})\|_{2}^{2} & =\left\|\sum_{j=1}^{d} \mathcal{B}_{j}\left(\boldsymbol{v}_{r_{j}}\right)\right\|_{2}^{2} \\
& \leq d \sum_{j=1}^{d}\left\|\mathcal{B}_{j}\left(\boldsymbol{v}_{r_{j}}\right)\right\|_{2}^{2} \\
& \left.\left.\leq 2 d \sum_{j=1}^{d}\left(\| \mathcal{B}_{j}\right]^{0_{j}}(\boldsymbol{v})\left\|_{2}^{2}+\right\| \mathcal{B}_{j}\right]_{0_{j}}(\boldsymbol{v}) \|_{2}^{2}\right) \\
& \leq 2 d\|\mathcal{M}(\boldsymbol{v})\|_{2}^{2} \\
& \leq 8 d \varepsilon^{2} .
\end{aligned}
$$

In light of Proposition 6 this completes the proof. 
Remark 7. The component operator $\mathcal{A}$ from the main theorem was not used at all in deriving properties (i) and (ii); on the other hand, only the measurements in $\mathcal{A}$ will be used to derive property (iii) from (i) and $(i i)$.

\section{A SOBOLEV INEQUALITY FOR INCOHERENT SUBSPACES}

The purpose of this section is to derive the following bound for signals lying near the null space of an incoherent matrix.

Theorem 8 (Sobolev inequality for incoherent subspaces). Let $d \geq 2$ and let $N=2^{n}$. Let $\mathcal{H}: \mathbb{C}^{N^{d}} \rightarrow$ $\mathbb{C}^{N^{d}}$ be the multivariate Haar wavelet transform. Let $\mathcal{B}: \mathbb{C}^{N^{d}} \rightarrow \mathbb{C}^{m}$ be a linear map with the property that $\mathcal{B H}^{*}: \mathbb{C}^{N^{d}} \rightarrow \mathbb{C}^{m}$ satisfies the restricted isometry property of order $2 s$ and level $\delta<1$. Then there is a universal constant $C>0$ such that for any signal $\boldsymbol{v} \in \mathbb{C}^{N^{d}}$ satisfying the tube constraint $\|\mathcal{B}(\boldsymbol{v})\|_{2} \leq \varepsilon$

$$
\|\boldsymbol{v}\|_{2} \leq C\left(\frac{\sqrt{b}\|\boldsymbol{v}\|_{T V}}{\sqrt{s}}\right) \log \left(N^{d}\right)+\varepsilon
$$

Note that Theorem 8 admits various corollaries for various families of random matrices with restricted isometries. For Gaussian random matrices, the theorem implies the following.

Corollary 9. Let $\mathcal{B}: \mathbb{C}^{N^{d}} \rightarrow \mathbb{C}^{m}$ be a linear map realizable as an $N^{d} \times m$ matrix whose entries are mean-zero i.i.d. Gaussian random variables. Then with probability exceeding $1-e^{-c m}$, the following bound holds for any $\boldsymbol{x} \in \mathbb{C}^{N^{d}}$ lying in the null-space of $\mathcal{B}$ :

$$
\|\boldsymbol{x}\|_{2} \lesssim\left(\frac{\sqrt{b}\|\boldsymbol{x}\|_{T V}}{\sqrt{m}}\right)\left[\log \left(N^{d}\right)\right]^{2} .
$$

Proof: From results on Gaussian matrices and the restricted isometry property (see e.g. [9], [36], [1], [46]), $\mathcal{B}$ satisfies the RIP of order $2 s$ and level $\delta<1$ with probability exceeding $1-e^{-c m}$ when $s$ is proportional to $m / \log \left(N^{d}\right)$. Substituting this value for $s$ into (27) yields the claim.

The proof of Theorem 8 goes as follows: as revealed through the proof of Proposition 6, any signal in the null space of an RIP matrix must be relatively flat, in that the norm of its best $s$-term approximation is bounded by the norm of the remaining terms. At the same time, Proposition 2 implies that the sequence of wavelet block-coefficient norms $\left\|\boldsymbol{c}_{(k)}\right\|_{2}$ in the null space of $\mathcal{B H}^{*}$ must be sufficiently compressible and bounded by the total-variation of $\boldsymbol{x}$. Combining these properties — flatness and tail-compressibility — produces the Sobolev-type inequality (27).

Proof of Theorem 8: 
Let $\boldsymbol{c}=\mathcal{H}(\boldsymbol{v}) \in \mathbb{C}^{N^{d}}$ represent the Haar transform of the signal error $\boldsymbol{v}=\boldsymbol{x}-\hat{\boldsymbol{x}}$. Suppose without loss of generality that the desired sparsity level $s$ is either smaller than $2^{d}-1$ or a positive multiple of $2^{d}-1$, and write $s=p\left(2^{d}-1\right.$ ) where either $p \in \mathbb{N}$ or $p \in(0,1)$ (for arbitrary $s \in \mathbb{N}$, we could consider $s^{\prime}=\left\lceil s /\left(2^{d}-1\right)\right\rceil$ which satisfies $\left.s^{\prime} \leq 2 s\right)$.

Let $S=S_{0} \subset[N]^{d}$ be the set of $s$ largest-magnitude entries of $\boldsymbol{c}$, let $S_{1}$ be the set of $s$ largestmagnitude entries of $\boldsymbol{c}$ in $[N]^{d} \backslash S_{0}$, and so on. Note that $c_{S}$ and similar expressions below can have both the meaning of restricting $c$ to the indices in $S$ as well as being the array whose entries are set to zero outside $S$.

By definition, $\left\|\boldsymbol{c}_{S_{0}}\right\|_{1}$ is at least as large as $\left\|\boldsymbol{c}_{\Omega}\right\|_{1}$ for any other $\Omega \subset[N]^{d}$ of cardinality $s$. Consequently, $\left\|\boldsymbol{c}_{S_{0}^{c}}\right\|_{1}$ is smaller than $\left\|\boldsymbol{c}_{\Omega^{c}}\right\|_{1}$ for any other $\Omega \subset[N]^{d}$ of cardinality s. Now, recall the alternative decomposition of $\boldsymbol{c}$ from Proposition 2 into blocks $\boldsymbol{c}_{(k)}$ of cardinality $2^{d}-1$, grouped according to the support of the corresponding wavelets. Because $s=p\left(2^{d}-1\right)$ for either $p \in \mathbb{N}$ or $p \in(0,1)$,

$$
\begin{aligned}
\left\|\boldsymbol{c}_{S_{0}^{c}}\right\|_{1} & =\sum_{k \geq 1}\left\|\boldsymbol{c}_{S_{k}}\right\|_{1} \\
& \leq \sum_{j \geq p+1}\left\|\boldsymbol{c}_{(j)}\right\|_{1} \\
& \leq\left(2^{d}-1\right)^{1 / 2} \sum_{j \geq p+1}\left\|\boldsymbol{c}_{(j)}\right\|_{2} \\
& \lesssim \sqrt{b}\|\boldsymbol{v}\|_{T V} \sum_{\ell=p+1}^{N^{d}} \frac{1}{\ell} \\
& \lesssim \sqrt{b}\|\boldsymbol{v}\|_{T V} \log \left(N^{d}\right),
\end{aligned}
$$

where the second to last inequality follows from Proposition 2 and the last inequality from properties of the geometric summation.

We use a similar procedure to bound the $\ell_{2}$-norm of the residual,

$$
\begin{aligned}
\left\|\boldsymbol{c}_{S_{0}^{c}}\right\|_{2}^{2} & \lesssim \sum_{j \geq p+1}\left\|\boldsymbol{c}_{(j)}\right\|_{2}^{2} \\
& \lesssim \frac{b\|\boldsymbol{v}\|_{T V}^{2}}{2^{d}} \sum_{\ell=p+1}^{N^{d}} \frac{1}{\ell^{2}} \\
& \lesssim \frac{\left(\sqrt{b}\|\boldsymbol{v}\|_{T V}\right)^{2}}{2^{d} \max (1, p)} \\
& \lesssim \frac{\left(\sqrt{b}\|\boldsymbol{v}\|_{T V}\right)^{2}}{s} .
\end{aligned}
$$


Then, $\left\|\boldsymbol{c}_{S_{0}^{c}}\right\|_{2} \lesssim\|\boldsymbol{v}\|_{T V} / \sqrt{s}$.

By assumption, $\boldsymbol{v}$ satisfies the tube constraint $\|\mathcal{B}(\boldsymbol{v})\|_{2} \leq \varepsilon$ and $\mathcal{B H}^{*}$ satisfies the restricted isometry property. We conclude that

$$
\begin{aligned}
\varepsilon & \geq\|\mathcal{B}(\boldsymbol{v})\|_{2}=\left\|\mathcal{B H}^{*}(\boldsymbol{c})\right\|_{2} \\
& \geq\left\|\mathcal{B H}^{*}\left(\boldsymbol{c}_{S_{0}}+\boldsymbol{c}_{S_{1}}\right)\right\|_{2}-\sum_{k=2}^{r}\left\|\mathcal{B H}^{*}\left(\boldsymbol{c}_{S_{k}}\right)\right\|_{2} \\
& \geq(1-\delta)\left\|\boldsymbol{c}_{S_{0}}+\boldsymbol{c}_{S_{1}}\right\|_{2}-(1+\delta) \sum_{k=2}^{r}\left\|\boldsymbol{c}_{S_{k}}\right\|_{2} \\
& \geq(1-\delta)\left\|\boldsymbol{c}_{S_{0}}\right\|_{2}-(1+\delta) \frac{1}{\sqrt{s}}\left\|\boldsymbol{c}_{S_{0}^{c}}\right\|_{1},
\end{aligned}
$$

the last inequality holding because the magnitude of each entry in the array $\boldsymbol{c}_{S_{k}}$ is smaller than the average magnitude of the entries in the array $\boldsymbol{c}_{S_{k-1}}$. Along with the tail bound (29), we can then conclude that, up to a constant in the restricted isometry level $\delta$,

$$
\left\|\boldsymbol{c}_{S_{0}}\right\|_{2} \lesssim \varepsilon+\log \left(N^{d}\right)\left(\frac{\sqrt{b}\|\boldsymbol{v}\|_{T V}}{\sqrt{s}}\right) .
$$

Combining this bound with the $\ell_{2}$-tail bound (30) and recalling that the Haar transform $\mathcal{H}: N^{d} \rightarrow N^{d}$ is an isometry,

$$
\|\boldsymbol{v}\|_{2}=\left\|\mathcal{H}^{*} \boldsymbol{c}\right\|_{2}=\|\boldsymbol{c}\|_{2} \leq\left\|\boldsymbol{c}_{S_{0}}\right\|_{2}+\left\|\boldsymbol{c}_{S_{0}^{c}}\right\|_{2} \lesssim \varepsilon+\log \left(N^{d}\right)\left(\frac{\sqrt{b}\|\boldsymbol{v}\|_{T V}}{\sqrt{s}}\right)
$$

which completes the proof.

\section{A. Proof of the Main Theorem}

Because we proved the bounds (i) and (ii) from the main theorem concerning stable gradient recovery in Section IV, it remains only to prove the signal recovery error bound (iii).

By feasibility of both $\boldsymbol{x}$ and $\hat{\boldsymbol{x}}$ for the constraint in the total variation minimization program, the signal error $\boldsymbol{v}=\boldsymbol{x}-\hat{\boldsymbol{x}}$ obeys the tube-constraint $\|\mathcal{A}(\boldsymbol{v})\|_{2} \leq 2 \varepsilon$. Applying Theorem 8 and the total variation 
bound (ii) yields

$$
\begin{aligned}
\|\boldsymbol{x}-\hat{\boldsymbol{x}}\|_{2} & =\|\boldsymbol{v}\|_{2} \\
& \lesssim \varepsilon+\log \left(N^{d}\right)\left(\frac{\sqrt{b}\|\boldsymbol{v}\|_{T V}}{\sqrt{s}}\right) \\
& \lesssim \varepsilon+\frac{\log \left(N^{d}\right) \sqrt{b}}{\sqrt{s}}\left(\left\|\boldsymbol{\nabla} \boldsymbol{x}-(\boldsymbol{\nabla} \boldsymbol{x})_{s}\right\|_{1 b}+\sqrt{s d} \varepsilon\right) \\
& =\log \left(N^{d}\right)\left(\sqrt{b d} \varepsilon+\frac{\sqrt{b}\left\|\boldsymbol{\nabla} \boldsymbol{x}-(\boldsymbol{\nabla} \boldsymbol{x})_{s}\right\|_{1 b}}{\sqrt{s}}\right) .
\end{aligned}
$$

The proof completes.

\section{APPENDIX}

\section{A. Derivation of Proposition 2}

Recall that the space $L_{p}(\Omega)(1 \leq p<\infty)$ for $\Omega \subset \mathbb{R}^{d}$ consists of all functions $f$ satisfying

$$
\|f\|_{L_{p}(\Omega)}=\left(\int_{\Omega}|f(u)|^{p} d u\right)^{1 / p}<\infty .
$$

The space $\operatorname{BV}(\Omega)$ of functions of bounded variation over the unit cube $Q=[0,1)^{d}$ is often used as a continuous model for natural images. Recall that a function $f \in L_{1}(Q)$ has finite bounded variation if and only if its distributional gradient is a bounded Radon measure, and this measure generates the BV seminorm $|f|_{B V(\Omega)}$. More precisely,

Definition 10. For a vector $\boldsymbol{v} \in \mathbb{R}^{d}$, we define the difference operator $\Delta_{\boldsymbol{v}}$ in the direction of $\boldsymbol{v}$ by

$$
\Delta_{\boldsymbol{v}}(f, \boldsymbol{x}):=f(\boldsymbol{x}+\boldsymbol{v})-f(\boldsymbol{x}) .
$$

We say that a function $f \in L_{1}(Q)$ is in $B V(Q)$ if and only if

$$
V_{Q}(f) \stackrel{\text { def }}{=} \sup _{h>0} h^{-1} \sum_{j=1}^{d}\left\|\Delta_{h \boldsymbol{e}_{j}}(f, \cdot)\right\|_{L_{1}\left(Q\left(h \boldsymbol{e}_{j}\right)\right)}=\lim _{h \rightarrow 0} h^{-1} \sum_{j=1}^{d}\left\|\Delta_{h \boldsymbol{e}_{j}}(f, \cdot)\right\|_{L_{1}\left(Q\left(h \boldsymbol{e}_{j}\right)\right)}<\infty
$$

where $\boldsymbol{e}_{j}$ denotes the $j$ th coordinate vector. The function $V_{Q}(f)$ provides a seminorm for $B V(Q)$ :

$$
|f|_{B V(Q)} \stackrel{\text { def }}{=} V_{Q}(f) \text {. }
$$

In particular, piecewise constant functions are in the space $B V(Q)$. For $N=2^{n}$ a power of two, recall the following relationship between the total-variation of a multidimensional signal $\boldsymbol{x} \in \mathbb{C}^{N^{d}}$ and the bounded variation of its isometric piecewise-constant representation $f \in \Sigma_{N} \subset L_{2}(Q)$ as defined in (10): 
Lemma 11. Let $N=2^{n}$ for some integral $n$. Let $\boldsymbol{x} \in \mathbb{C}^{N^{d}}$ and let $f \in \Sigma_{N}$ be its isometric embedding as in (10). Then $|f|_{B V} \leq N^{-d / 2+1} \sqrt{b}\|\boldsymbol{x}\|_{T V}$.

Proof: For $h<\frac{1}{N}$,

$$
\Delta_{h e_{k}}(f, \boldsymbol{u})= \begin{cases}N^{d / 2}\left(\boldsymbol{x}_{\ell^{(k)}}-\boldsymbol{x}_{\ell}\right) & \frac{\ell_{i}}{N}-h \leq u_{i} \leq \frac{\ell_{i}}{N} \\ 0, & \text { else }\end{cases}
$$

where

$$
\ell_{i}^{(k)}= \begin{cases}\ell_{i} & i \neq k \\ \ell_{i}+1, & i=k\end{cases}
$$

Thus

$$
\begin{aligned}
|f|_{B V} & =\lim _{h \rightarrow 0} \frac{1}{h} \sum_{k=1}^{d}\left[\int_{0}^{1} \int_{0}^{1} \ldots \int_{0}^{1}\left|f\left(\boldsymbol{u}+h \boldsymbol{e}_{\boldsymbol{k}}\right)-f(\boldsymbol{u})\right| d \boldsymbol{u}\right] \\
& =\sum_{k=1}^{d} N^{d / 2}\left[\sum_{\boldsymbol{\ell}} \frac{1}{N^{d-1}}\left|\boldsymbol{x}_{\ell^{(k)}}-\boldsymbol{x}_{\ell}\right|\right] \\
& \leq N^{-d / 2+1}\|\boldsymbol{\nabla} \boldsymbol{x}\|_{1} \\
& \leq N^{-d / 2+1} \sqrt{b}\|\boldsymbol{x}\|_{T V} .
\end{aligned}
$$

Cohen, Dahmen, Daubechies, and DeVore showed in [15] that the properly normalized sequence of rearranged wavelet coefficients associated to a function $f \in L_{2}(\Omega)$ of bounded variation is in weak- $\ell_{1}$, and its weak- $\ell_{1}$ seminorm is bounded by the function BV seminorm. Using different normalizations to those used in [15] — we use the $L_{2}$-normalization for the Haar wavelets as opposed to the $L_{1}$-normalization - we consider the Haar wavelet coefficients $f_{I}^{e}=\left\langle f, h_{I}^{e}\right\rangle$ and consider the wavelet coefficient block $f_{I}=\left(f_{I}^{e}\right)_{e \in E} \in \mathbb{C}^{2^{d}-1}$ associated to those Haar wavelets supported on the dyadic cube $I$. With this notation, Theorem 1.1 of [15] applied to the Haar wavelet system over $L_{2}(Q)$ reads:

Proposition 12. Let $d \geq 2$. Then there exists a constant $C>0$ such that the following holds for all $f \in B V(Q)$. Let the wavelet coefficient block with kth largest $\ell_{2}$-norm be denoted by $f_{(k)}$, and suppose that this block is associated to the dyadic cube $I_{j, k}$ with side-length $2^{-j}$. Then

$$
\left\|f_{(k)}\right\|_{2} \leq C \frac{2^{j(d-2) / 2}|f|_{B V}}{k} .
$$


Proposition 2 results by translating Proposition 12 to the discrete setting of $\mathbb{C}^{N^{d}}$ using the isometry (10) and Lemma 11. We note that a stronger version of this result was provided for the 2-dimensional Haar wavelet basis in [16] and used in the proof of stable image recovery from total-variation minimization in [37].

\section{B. Proof of Proposition 6}

We prove Proposition 6 here for the isotropic case, $b=d$. The complete proof of the anisotropic case $b=1$ can be found in [37], and all other cases can be proven similarly.

Assume that the cone and tube constraints are in force. As in the proof of the cone constraint, let $R_{1} \subset R^{c}$ contain the next $4 s$ largest blocks of $\boldsymbol{h}$ on $R^{c}, R_{2}$ the next $4 s$ largest, and so on. We write $\boldsymbol{h}_{R_{j}}$ to mean the array $\boldsymbol{h}$ restricted to its elements indexed by $R_{j}$, and write $\boldsymbol{h}_{\boldsymbol{\alpha}}$ to denote the array $\left(\boldsymbol{h}_{(\ell, \boldsymbol{\alpha})}\right)_{\ell=1}^{d}$. Thus each set satisfies $\left|R_{i}\right|=4 s b$ (except possibly the last set), and $\left(\boldsymbol{h}_{\boldsymbol{\alpha}, \ell}\right)_{(\boldsymbol{\alpha}, \ell) \in R_{j+1}}$ has $\ell_{2}$-norm smaller than the average of all blocks of $\boldsymbol{h}_{R_{j}}$ :

$$
\left\|\boldsymbol{h}_{\boldsymbol{\alpha}}\right\|_{2} \leq \frac{1}{4 s} \sum_{\boldsymbol{\beta} \in R_{j}}\left\|\boldsymbol{h}_{\boldsymbol{\beta}}\right\|_{2}=\frac{1}{4 s}\left\|\boldsymbol{h}_{R_{j}}\right\|_{1,2} \quad \text { for all } \boldsymbol{\alpha} \in R_{j+1} .
$$

Therefore we have,

$$
\begin{aligned}
\left\|\boldsymbol{h}_{R_{j+1}}\right\|_{2}^{2} & =\sum_{\boldsymbol{\alpha} \in R_{j+1}}\left\|\boldsymbol{h}_{\boldsymbol{\alpha}}\right\|_{2}^{2} \\
& \leq \sum_{\boldsymbol{\alpha} \in R_{j+1}} \frac{1}{(4 s)^{2}}\left\|\boldsymbol{h}_{R_{j}}\right\|_{1,2}^{2} \\
& =\frac{1}{4 s}\left\|\boldsymbol{h}_{R_{j}}\right\|_{1,2}^{2} .
\end{aligned}
$$

Combining this with the cone constraint yields 


$$
\begin{aligned}
\sum_{j \geq 2}\left\|\boldsymbol{h}_{R_{j}}\right\|_{2} & \leq \frac{1}{\sqrt{4 s}} \sum_{j \geq 1}\left\|\boldsymbol{h}_{R_{j}}\right\|_{1,2} \\
& =\frac{1}{\sqrt{4 s}} \sum_{\boldsymbol{\alpha} \in R^{c}}\left\|\boldsymbol{h}_{\boldsymbol{\alpha}}\right\|_{2} \\
& =\frac{1}{\sqrt{4 s}}\left\|\boldsymbol{h}_{R^{c}}\right\|_{1,2} \\
& \leq \frac{1}{\sqrt{4 s}}\left\|\boldsymbol{h}_{R}\right\|_{1,2}+\frac{1}{\sqrt{4 s}} \sigma \\
& \leq \frac{1}{2}\left\|\boldsymbol{h}_{R}\right\|_{2}+\frac{1}{\sqrt{4 s}} \sigma
\end{aligned}
$$

where in the last line we have utilized the fact that $\left\|\boldsymbol{h}_{R}\right\|_{1,2} \leq \sqrt{s}\left\|\boldsymbol{h}_{R}\right\|_{2}$. Next by the tube constraint we have

$$
\begin{aligned}
\sqrt{2 d} \varepsilon & \geq\|\mathcal{B} \boldsymbol{h}\|_{2} \\
& \geq \sqrt{1-\delta}\left\|\boldsymbol{h}_{R}+\boldsymbol{h}_{R_{1}}\right\|_{2}-\sqrt{1+\delta} \sum_{j \geq 2}\left\|\boldsymbol{h}_{R_{j}}\right\|_{2} \\
& \geq \sqrt{1-\delta}\left\|\boldsymbol{h}_{R}+\boldsymbol{h}_{R_{1}}\right\|_{2}-\sqrt{1+\delta}\left(\frac{1}{2}\left\|\boldsymbol{h}_{R}\right\|_{2}+\frac{1}{\sqrt{4 s}} \sigma\right) \\
& \geq\left(\sqrt{1-\delta}-\frac{1}{2} \sqrt{1+\delta}\right)\left\|\boldsymbol{h}_{R}+\boldsymbol{h}_{R_{1}}\right\|_{2}-\sqrt{1+\delta}\left(\frac{1}{\sqrt{4 s}} \sigma\right) .
\end{aligned}
$$

Using the fact that $\delta<1 / 3$, this implies that

$$
\left\|\boldsymbol{h}_{R}+\boldsymbol{h}_{R_{1}}\right\|_{2} \leq 10 \sqrt{d} \varepsilon+\frac{3}{\sqrt{s}} \sigma .
$$

The bound (23) then follows since

$$
\begin{aligned}
\|\boldsymbol{h}\|_{2} & \leq\left\|\boldsymbol{h}_{R}+\boldsymbol{h}_{R_{1}}\right\|_{2}+\sum_{j \geq 2}\left\|\boldsymbol{h}_{R_{j}}\right\|_{2} \\
& \leq\left\|\boldsymbol{h}_{R}+\boldsymbol{h}_{R_{1}}\right\|_{2}+\frac{1}{2}\left\|\boldsymbol{h}_{R}\right\|_{2}+\frac{1}{\sqrt{4 s}} \sigma \\
& \lesssim \sqrt{d} \varepsilon+\frac{1}{\sqrt{s}} \sigma .
\end{aligned}
$$


Similarly, the bound (24) follows from the cone constraint,

$$
\begin{aligned}
\|\boldsymbol{h}\|_{1,2} & \leq 2\left\|\boldsymbol{h}_{R}\right\|_{1,2}+\sigma \\
& \leq 2 \sqrt{s}\left\|\boldsymbol{h}_{R}\right\|_{2}+\sigma \\
& \lesssim \sqrt{s}\left(\sqrt{d} \varepsilon+\frac{1}{\sqrt{s}} \sigma\right)+\sigma \\
& =\sqrt{s d} \varepsilon+\sigma
\end{aligned}
$$

which completes the proof.

\section{Acknowledgment}

We would like to thank John Doyle, Christina Frederick, and Mark Tygert for helpful improvements and insights. We would also like to thank the reviewers of the manuscript for their thoughtful suggestions which significantly improved the manuscript.

\section{REFERENCES}

[1] R. G. Baraniuk, M. Davenport, R. A. DeVore, and M. Wakin. A simple proof of the Restricted Isometry Property for random matrices. Constr. Approx., 28(3):253-263, 2008.

[2] F. Baus, M. Nikolova, and G. Steidl. Fully smoothed $\ell_{1}-$ TV models: Bounds for the minimizers and parameter choice. $J$. Math. Imaging Vis., pages 1-13, 2013.

[3] S. Boyd and L. Vandenberghe. Convex optimization. Cambridge university press, 2004.

[4] M. Burger and S. Osher. Convergence rates of convex variational regularization. Inverse probl., 20(5):1411, 2004.

[5] N. Burq, S. Dyatlov, R. Ward, and M. Zworski. Weighted eigenfunction estimates with applications to compressed sensing. arXiv:1111.2383, 2012.

[6] E. Candès, Y. Eldar, D. Needell, and P. Randall. Compressed sensing with coherent and redundant dictionaries. Appl. Comput. Hamon. A., 31(1):59-73, 2011.

[7] E. Candès and J. Romberg. Signal recovery from random projections. In Proc. SPIE Conference on Computational Imaging III, volume 5674, pages 76-86. SPIE, 2005.

[8] E. Candès, J. Romberg, and T. Tao. Stable signal recovery from incomplete and inaccurate measurements. Communications on Pure and Applied Mathematics, 59(8):1207-1223, 2006.

[9] E. Candès and T. Tao. Decoding by linear programming. IEEE Trans. Inform. Theory, 51:4203-4215, 2005.

[10] E. Candès and T. Tao. Near optimal signal recovery from random projections: Universal encoding strategies? IEEE Trans. Inform. Theory, 52(12):5406-5425, 2006.

[11] E. Candès, T. Tao, and J. Romberg. Robust uncertainty principles: exact signal reconstruction from highly incomplete frequency information. IEEE Trans. Inform. Theory, 52(2):489-509, 2006.

[12] V. Caselles, A. Chambolle, and M. Novaga. The discontinuity set of solutions of the tv denoising problem and some extensions. Multiscale Model. Sim., 6(3):879-894, 2007. 
[13] V. Caselles, A. Chambolle, and M. Novaga. Regularity for solutions of the total variation denoising problem. Rev. Mat. Iberoam., 27(1):233-252, 2011.

[14] T.F. Chan, J. Shen, and H.M. Zhou. Total variation wavelet inpainting. J. Math. Imaging Vis., 25(1):107-125, 2006.

[15] A. Cohen, W. Dahmen, I. Daubechies, and R. DeVore. Harmonic analysis of the space BV. Revistaua Matematica Iberoamericana, 19(1):235-263, 2003.

[16] A. Cohen, R. DeVore, P. Petrushev, and H. Xu. Nonlinear approximation and the space $B V\left(\mathbb{R}^{2}\right)$. Am. J. of Math, 121:587-628, 1999.

[17] Patrick L Combettes and Jean-Christophe Pesquet. Proximal splitting methods in signal processing. Fixed-Point Algorithms for Inverse Problems in Science and Engineering, pages 185-212, 2011.

[18] Compressed sensing webpage. http://www.dsp.ece.rice.edu/cs/.

[19] I. Daubechies. Ten Lectures on Wavelets. SIAM, 1992.

[20] F. Dibos and G. Koepfler. Global total variation minimization. SIAM J.Numer. Anal., 37(2):646-664, 1999.

[21] D. Donoho and P. Stark. Uncertainty principles and signal recovery. SIAM J. on Appl. Math., pages 906-931, 1989.

[22] D. L. Donoho. Compressed sensing. IEEE T. Inform. Theory, 52(4):1289-1306, 2006.

[23] A. Garnaev and E. Gluskin. On widths of the Euclidean ball. Sov. Math. Dokl., 30:200-204, 1984.

[24] M. Grasmair. Locally adaptive total variation regularization. Lect. Notes Comput. SC, pages 331-342, 2009.

[25] B. Kai Tobias, U. Martin, and F. Jens. Suppression of MRI truncation artifacts using total variation constrained data extrapolation. Int. J. Biomedical Imaging, 2008.

[26] B. Kashin. The widths of certain finite dimensional sets and classes of smooth functions. Izvestia, 41:334-351, 1977.

[27] S.L. Keeling. Total variation based convex filters for medical imaging. Appl. Math. Comput., 139(1):101-119, 2003.

[28] F. Krahmer, S. Mendelson, and H. Rauhut. Suprema of chaos processes and the restricted isometry property. arxiv:1207.0235, 2012.

[29] F. Krahmer and R. Ward. New and improved Johnson-Lindenstrauss embeddings via the Restricted Isometry Property. SIAM J. Math. Anal., 43(3):1269-1281, 2011.

[30] Felix Krahmer and Rachel Ward. Beyond incoherence: stable and robust sampling strategies for compressive imaging. 2012. Submitted.

[31] Y. Liu and Q. Wan. Total variation minimization based compressive wideband spectrum sensing for cognitive radios. Submitted, 2011.

[32] Yulong Liu, Tiebin Mi, and Shidong Li. Compressed sensing with general frames via optimal-dual-based $\ell_{1}$-analysis. IEEE T. Inform. Theory, 58(7):4201-4214, 2012.

[33] M. Lustig, D. Donoho, and J.M. Pauly. Sparse MRI: The application of compressed sensing for rapid MRI imaging. Magnetic Resonance in Medicine, 58(6):1182-1195, 2007.

[34] M. Lustig, D. Donoho, J. Santos, and J. Pauly. Compressed sensing MRI. IEEE Sig. Proc. Mag., 25(2):72-82, 2008.

[35] S. Ma, W. Yin, Y. Zhang, and A. Chakraborty. An efficient algorithm for compressed MR imaging using total variation and wavelets. In IEEE Conf. Comp. Vision Pattern Recog., 2008.

[36] S. Mendelson, A. Pajor, and N. Tomczak-Jaegermann. Uniform uncertainty principle for Bernoulli and subgaussian ensembles. Constr. Approx., 28(3):277-289, 2008.

[37] D. Needell and R. Ward. Stable image reconstruction using total variation minimization. SIAM J. Imaging Sciences, 2012. To appear. 
[38] B. Nett, J. Tang, S. Leng, and G.H. Chen. Tomosynthesis via total variation minimization reconstruction and prior image constrained compressed sensing (PICCS) on a C-arm system. In Proc. Soc. Photo-Optical Instr. Eng., volume 6913. NIH Public Access, 2008.

[39] M. Nikolova, Y. Wen, and R. Chan. Exact histogram specification for digital images using a variational approach. Technical report, Tech. Report, HAL-00723448, 2012.

[40] S. Osher, M. Burger, D. Goldfarb, J. Xu, and W. Yin. An iterative regularization method for total variation-based image restoration. Multiscale Model. Sim., 4(2):460-489, 2005.

[41] S. Osher, A. Solé, and L. Vese. Image decomposition and restoration using total variation minimization and the $\mathrm{H}^{-1}$ norm. Multiscale Model. Sim., 1:349-370, 2003.

[42] H. Rauhut. Compressive Sensing and Structured Random Matrices. In M. Fornasier, editor, Theoretical Foundations and Numerical Methods for Sparse Recovery, volume 9 of Radon Series Comp. Appl. Math., pages 1-92. deGruyter, 2010.

[43] H. Rauhut and R. Ward. Sparse recovery for spherical harmonic expansions. Proc. SampTA, 2011.

[44] H. Rauhut and R. Ward. Sparse Legendre expansions via $\ell_{1}$-minimization. Journal of Approximation Theory, 164:517-533, 2012.

[45] W. Ring. Structural properties of solutions to total variation regularization problems. ESAIM-Math. Model. Num., 34(04):799-810, 2000.

[46] M. Rudelson and R. Vershynin. On sparse reconstruction from Fourier and Gaussian measurements. Comm. Pure Appl. Math., 61:1025-1045, 2008.

[47] L.I. Rudin, S. Osher, and E. Fatemi. Nonlinear total variation based noise removal algorithms. Physica D: Nonlinear Phenomena, 60(1-4):259-268, 1992.

[48] S. Vaiter, G. Peyr'e, C. Dossal, and J. Fadili. Robust sparse analysis regularization. IEEE T. Inform. Theory, 2012. To appear.

[49] J. Yuan, C. Schnörr, and G. Steidl. Total-variation based piecewise affine regularization. In X.-C. Tai, K. Mórken, M. Lysaker, and K.-A. Lie, editors, Lect. Notes Comput. SC, volume 5567 of LNCS, pages 552-564. Springer, 2009. 